\title{
Genome-Wide Identification and Transferability of Microsatellite Markers between Palmae Species
}

\section{OPEN ACCESS}

Edited by:

Maoteng Li,

Huazhong University of Science and

Technology, China

Reviewed by:

Changying Zeng,

Institute of Tropical Biosciences and

Biotechnology, China

Jiaqin Shi,

Chinese Academy of Agricultural

Sciences, China

${ }^{*}$ Correspondence:

Yong Xiao

xiaoyong1980@catas.cn

Xintao Lei

xtlei@126.com

${ }^{\dagger}$ These authors have contributed equally to this work.

Specialty section:

This article was submitted to Crop Science and Horticulture, a section of the journal

Frontiers in Plant Science

Received: 04 August 2016 Accepted: 06 October 2016 Published: 25 October 2016

Citation:

Xiao Y, Xia W, Ma J, Mason AS, Fan $H$, Shi P, Lei X, Ma Z and Peng $M$ (2016) Genome-Wide Identification and Transferability of Microsatellite Markers between Palmae Species.

Front. Plant Sci. 7:1578. doi: 10.3389/fpls.2016.01578

\begin{abstract}
Yong Xiao ${ }^{1 * t}$, Wei Xia ${ }^{1,2+}$, Jianwei Ma ${ }^{1}$, Annaliese S. Mason ${ }^{3}$, Haikuo Fan ${ }^{1}$, Peng Shi ${ }^{1}$, Xintao Lei ${ }^{1 *}$, Zilong $\mathrm{Ma}^{4}$ and Ming Peng ${ }^{4}$

${ }^{1}$ Hainan Key Laboratory of Tropical Oil Crops Biology/Coconut Research Institute, Chinese Academy of Tropical Agricultural Sciences, Wenchang, China, ${ }^{2}$ College of Agriculture, Hainan University, Haikou, China, ${ }^{3}$ Department of Plant Breeding, IFZ Research Centre for Biosystems, Land Use and Nutrition, Justus Liebig University Giessen, Giessen, Germany, ${ }^{4}$ Institute of Tropical Bioscience and Biotechnology, Chinese Academy of Tropical Agricultural Science, Haikou, China
\end{abstract}

The Palmae family contains 202 genera and approximately 2800 species. Except for Elaeis guineensis and Phoenix dactylifera, almost no genetic and genomic information is available for Palmae species. Therefore, this is an obstacle to the conservation and genetic assessment of Palmae species, especially those that are currently endangered. The study was performed to develop a large number of microsatellite markers which can be used for genetic analysis in different Palmae species. Based on the assembled genome of $E$. guineensis and $P$. dactylifera, a total of 814383 and 371629 microsatellites were identified. Among these microsatellites identified in E. guineensis, 734509 primer pairs could be designed from the flanking sequences of these microsatellites. The majority (618 762) of these designed primer pairs had in silico products in the genome of $E$. guineensis. These 618762 primer pairs were subsequently used to in silico amplify the genome of $P$. dactylifera. A total of 7265 conserved microsatellites were identified between $E$. guineensis and $P$. dactylifera. One hundred and thirty-five primer pairs flanking the conserved SSRs were stochastically selected and validated to have high cross-genera transferability, varying from 16.7 to $93.3 \%$ with an average of $73.7 \%$. These genome-wide conserved microsatellite markers will provide a useful tool for genetic assessment and conservation of different Palmae species in the future.

Keywords: palm, Elaeis guineensis, Phoenix dactylifera, microsatellite, cross-genera transferability

\section{INTRODUCTION}

Palmae is a monocot family containing 202 genera and approximately 2800 species mainly distributed in tropical and subtropical regions. Palm trees generally have a solitary shoot bearing a crop of very large leaves. The majority of palm trees are used as landscape plants, planted along both sides of the street. However, there are also some important economic crops in this family, such as Elaeis guineensis, Phoenix dactylifera, Cocos nucifera and Areca catechu. Of the four economic crops, the African oil palm E. guineensis has the largest plantation area (17 million hectares), producing 50 million tons of palm oil annually. Due to its economic importance, the whole genome sequence of E. guineensis has already been completed and was released in August 2013 (Singh et al., 2013). The draft genome sequence of related species $P$. dactylifera has also been reported (AI-Dous et al., 2011; AI-Mssallem et al., 2013). The genome sizes of E. guineensis and P. dactylifera are 1.535 
and $605 \mathrm{Mb}$, respectively. However, only $657.86 \mathrm{Mb}$ (42.86\%) can be mapped into the 16 assembled chromosomes of E. guineensis, and some chromosomes are not assembled in $P$. dactylifera. Previous research based on phylogenetic dating has shown a divergence 65 Mya between E. guineensis and P. dactylifera (Singh et al., 2013). Except for these species, other Palmae are still understudied and few genetic and genomic resources are available. This is an obstacle to the conservation and genetic assessment of Palmae species, especially those that are currently endangered.

Microsatellites (simple sequence repeats, SSRs) are tandem DNA repeats which are mostly distributed throughout noncoding regions of eukaryotic genomes. Microsatellite markers are generally co-dominant and high polymorphic, and are widely used for constructing genetic maps, diversity analysis and quantitative trait locus mapping (Qiu et al., 2006; Xiao et al., 2010; Zhang et al., 2012; Xia et al., 2014). Due to lack of selection pressure on these non-coding sequences, microsatellites are generally regarded as neutral genetic markers. Recently, microsatellites have been also validated to have important biological functions, such as the regulation of chromatin organization, DNA metabolic processes, gene activity and RNA structure (Li et al., 2002, 2004; Gemayel et al., 2010). Some research has already been done to identify microsatellite markers via probe hybridization against genomic/cDNA clones (Rivera et al., 1999). This method of developing microsatellites is still low efficiency and high cost. With the use of next-generation sequencing (NGS) technology, it became possible to develop large numbers of molecular markers through in silico analysis of available bacterial artificial chromosome (BAC) sequences (Xu et al., 2010), BAC-end sequences (BES) (Bohra et al., 2011), genome survey sequences (WGSs) (Marek et al., 2001), expressed sequence tags (Low et al., 2008), and whole genome shotgun sequences (WGSs) (Shi et al., 2013). In the Palmae family, cDNA library tags and transcriptome data have been used to develop EST-SSRs in E. guineensis (Xiao et al., 2014), P. dactylifera (Zhao et al., 2012), and C. nucifera (Xiao et al., 2014). With the release of the whole genome sequences of $E$. guineensis and P. dactylifera, this will provide an opportunity to develop larger number of microsatellite markers and ascertain their distribution across different chromosomes in the two Palmae species. However, except for these two Palmae species, identification of microsatellite markers is difficult because of the lack or scarcity of available sequence data in other Palmae species.

It has been well recognized that some molecular markers (including microsatellite markers) can be transferable from different genotypes within or between species or even between genera (Wang et al., 2012). Such interspecific or intergeneric transferability for markers will provide some useful genetic information for poorly-studied related species, contributing to conservation, genetic assessment, and construction of linkage maps. Meanwhile, the transferability of microsatellite markers depends on the genetic relationship between the species. Mnejja et al. (2010) reported that microsatellite markers developed from peach and almond had a higher transferability rate to other Prunus species than to three other non-Prunus rosaceous genera (Mnejja et al., 2005). In previous research, microsatellite cross-species transferability is identified generally via PCR amplification in different related species using primer pairs designed from one species (Decroocq et al., 2003; Gasic et al., 2009). This method to identify cross-species transferability of primer pairs is very low efficiency. Meanwhile, the number of primer pairs with cross-species transferability identified is also limited.

Among the Palmae family, the whole genome sequences of $E$. guineensis and P. dactylifera have been completed and released. This study (1) identified a large number of microsatellites and analyzed the frequency and distribution of microsatellites in the two palm species, respectively; (2) analyzed the distribution of these microsatellites in 16 chromosomes of E. guineensis; (3) identified conversed microsatellites genome-wide between E. guineensis and P. dactylifera; (4) validated the cross-genera transferability of these conserved microsatellites in Palmae.

\section{MATERIALS AND METHODS}

\section{Plant Materials and DNA Extraction}

Thirty-two samples from different palm species were collected from the Palmae germplasm nursery of the Coconut Research Institute in Wenchang, Hainan, China: Chrysalldocarpus lutescens, Livistona australis, Dictyosperma Album, Cary mitis, Corypha umbraculifea, Latanic lontaroides, Phoenix loureirii, Cyrtostachys renda, Veitchia merrillii, Hyophorbe verschaffeltii, Chrysalidocarpus lucubensis, Sabal palmetto, Wodyetia bifurcate, Trachycarpus nana, Areca triandra, Rhapis excels, Butia capitata, Cryosophila albida, Chamaedorea Elegans, Pritchardia pacifica, Dypsis decaryi, Phoenix robusta, Arenga engieri, Borassus flabellifer, Bismarckia Hildebr, C. nucifera, E. guineensis, A. catechu, Chamaerops ritchieana, Iraq candy date, $P$. dactylifera, and Hyophorbe lagenicaulis. Detailed information for these individual species is listed in Figure S1. DNA samples were prepared from young leaves of the 32 palm species samples using the mini-CTAB method (Stewart and Via, 1993).

\section{Sources of Genome Sequences and Gene Annotation Information}

The genomic sequences from E. guineensis and P. dactylifera were downloaded from the National Center of Biotechnology Information (NCBI). A total of 1056.95, 1038.22, and 506.67 Mb from 202 467, 219 249, and 143380 contigs were used for microsatellite identification in E. guineensis and P. dactylifera, respectively.

A total of 30057 genes were predicted by the National Center for Biotechnology information in E. guineensis. The gene annotation information was downloaded from NCBI. The mining of transposable elements (TEs) in E. guineensis genome was done using RepeatMasker (Smit et al., 2013/2015) software with the TE database of Arabidopsis and rice.

\section{Identification of Putative SSRs and Primer Design}

The SSR analysis software Msatfinder (https://github.com/ knirirr/Msatfinder) was used to identify all possible mono, di-, tri-, tetra, penta- ,and hexa-nucleotide SSRs with a minimum set of $12,4,4,4,4$, and 4 repeats, respectively (Thurston and 
Field, 2005). Subsequently, primer pairs were designed based on the sequences flanking the SSRs using Primer 3 software (Rozen and Skaletsky, 1999), with melting temperatures $58-62^{\circ} \mathrm{C}$, primer lengths 18-24 bp, GC content 45-55\%, expected fragment size 100-250 bp.

\section{ePCR}

A total of 734509 primer pairs were designed based on the flanking sequences of 814383 microsatellites of E. guineensis with primer 3 . These primer pairs were used to in silico amplify the genomic sequences of $P$. dactylifera using the ePCR software (www.ncbi.nlm.nih.gov/tools/epcr) (Schuler, 1997; Rotmistrovsky et al., 2004). The primer nucleotide mismatch allowed was no more than $1 \mathrm{nt}$. Meanwhile, the size difference allowed between the PCR products was less than 100 bp between the in silico PCR product in P. dactylifera and the expected PCR product in E. guineensis.

\section{PCR Amplification and Electrophoresis}

PCR amplifications were performed in $10 \mathrm{ul}$ reaction mixtures containing 100 ng genomic DNA, $1 \times$ PCR buffer, $2 \mathrm{mM} \mathrm{MgCl}_{2}$, $1 \mathrm{U}$ Taq DNA polymerase (TakaRa, China), $0.5 \mathrm{uM}$ of each primer and $0.2 \mathrm{mM} \mathrm{dNTP}$ mix, with the following program: denaturation for $4 \mathrm{~min}$ at $94^{\circ} \mathrm{C}, 30$ cycles of $30 \mathrm{~s}$ at $94^{\circ} \mathrm{C}, 30 \mathrm{~s}$ at $56^{\circ} \mathrm{C}$ and $30 \mathrm{~s}$ at $72^{\circ} \mathrm{C}$ for elongation, with a final extension of 7 min at $72^{\circ} \mathrm{C}$. PCR products were electrophoretically separated on $1 \%$ agarose gels. Product sizes were determined by comparison with a 100 bp DNA ladder.

\section{RESULTS}

\section{Frequency and Distribution of Simple Sequence Repeats in Elaeis guineensis and Phoenix dactylifera}

A total of 814383 and 371629 mononucleotide to hexanucleotide repeat microsatellite sequences were identified from 1057 and $507 \mathrm{Mb}$ of assembled genomic sequences of E. guineensis and $P$. dactylifera, respectively (Table 1), with an average of 770.4 and 733 microsatellites per $\mathrm{Mb}$, or one microsatellite per 1.29 and $1.36 \mathrm{~Kb}$.

Comparative analysis showed that the motif frequencies of microsatellites in the assembled genomic sequences of $E$. guineensis and $P$. dactylifera were almost identical (Figure 1). Among these identified microsatellites, dinucleotide motif types were the most abundant, comprising 76.6 and $72.7 \%$ of total motifs in E. guineensis and P. dactylifera. Trinucleotide motifs comprised the next largest proportion (10.4 and $13.2 \%$ in E. guineensis and $P$. dactylifera respectively), followed by mononucleotide motifs (9.6 and 9.7\%). Smaller frequencies of tetranucleotide (2.3 and 3.2\%), pentanucleotide (0.8 and $0.8 \%$ ), and hexanucleotide $(0.3$ and $0.3 \%)$ motifs were also observed. The dominant motif types were all $\mathrm{A} / \mathrm{T}$ in the two palm species, accounting for 95.3 and $93.2 \%$ of the total mononucleotide repeats, respectively. However, for dinucleotide motifs, the dominant/major motif was "AT" in E. guineensis, accounting for $23.8 \%$ of the total dinucleotide repeats, while the dominant/major motif was CT/AG in P. dactylifera, accounting for $24.4 \%$ of the total dinucleotide repeats. The trinucleotide motifs were relatively diverse. Among them, TCT/AGA was the richest repeat type in the tri-motifs of E. guineensis, accounting for $15.5 \%$ of total trinucleotide motifs in the species. However, in $P$. dactylifera, TTC/GAA and TTA/TAA were the dominant motifs, accounting for $11.3 \%$ of total trinucleotide motifs. Comparative analysis of motif type showed that the dominant motifs were all $\mathrm{A} / \mathrm{T}$ rich, whereas the absent/scarce motifs were mostly $\mathrm{C} / \mathrm{G}$ rich, which is in accordance with previous research results from Brassica species (Shi et al., 2013, Figure 2). Meanwhile, the distributions with respect to the motif repeat number of microsatellites in the assembled genome of the two palm species were almost identical. With increasing motif repeat number, the number of microsatellites with that motif significantly decreased (Figure 2).

\section{Genomic Distribution}

The genome-wide distributions of microsatellites and their relation with the annotated genome components (including genes and TEs) were investigated based on the assembled genomic sequences of E. guineensis. A total of 814383 microsatellites were identified in the assembled sequences of E. guineensis. Among these identified microsatellites, only 447 $244(54.92 \%)$ could be mapped to the 16 chromosomes of E. guineensis. The distribution of the mapped microsatellites across the different chromosomes was uneven (Table 2). A higher microsatellite density was found in chromosome 1 (723.71 microsatellites per $\mathrm{Mb}$ ), chromosome 2 (707.23 microsatellites per $\mathrm{Mb}$ ), chromosome 10 (724.4 microsatellites per $\mathrm{Mb}$ ), and chromosome 19 (735.56 microsatellites per $\mathrm{Mb}$ ) compared to the other chromosomes. Gene density was also comparatively high in these four chromosomes. Meanwhile, the frequency of microsatellites was high near both ends but low near the middle of all the chromosomes (Figure 3 and Figure S2): microsatellites had high density in chromosome peri-telomeres. The distribution of microsatellites was highly correlated with the distribution of annotated genes, but negatively correlated with the distribution of annotated TEs (Figure 3 and Table 2).

\section{Copy Number of these Identified Microsatellites in Genome of Elaeis guineensis}

A total of 734509 primer pairs were successfully designed from the flanking sequences of mono- to hexanucleotide microsatellites of E. guineensis. Primer pairs could not be designed for the remaining microsatellites, mainly due to lack of sufficient flanking sequence from both sides of the identified microsatellites.

Subsequently, these designed primer pairs were used to in silico amplify the assembled genome of E. guineensis. A total of 618762 primer pairs had in silico products. Meanwhile, in these primer pairs, 419958 (67.87\%), 45544 (7.36\%), 19093 (3.09\%), and 134167 (21.68\%) could generate 1, 2, 3, and >3 in silico PCR products, respectively, from the assembled genomic sequence of E. guineensis. A total of 9687176 in silico fragments could be generated based on these designed primer pairs. Among 
TABLE 1 | Number, repeat number and total length of the mononucleotide to hexanucleotide motifs of microsatellites in the assembled genomic sequences of Elaeis guineensis and Phoenix dactylifera.

\begin{tabular}{|c|c|c|c|c|c|c|}
\hline \multirow[t]{2}{*}{ Motif } & \multicolumn{3}{|c|}{ Elaeis guineensis } & \multicolumn{3}{|c|}{ Phoenix dactylifera } \\
\hline & Number & Repeat number (average) & Total length & Number & Repeat number & Total length \\
\hline Mono & 78184 (9.6\%) & $12-51(13.632)$ & 1065826 & 35865 (9.7\%) & $12-115$ (13.509) & 484504 \\
\hline $\mathrm{a} / \mathrm{t}$ & 74526 (9.2\%) & $12-51(13.634)$ & 1016054 & 31302 (8.4\%) & $12-80(13.422)$ & 420148 \\
\hline $\mathrm{c} / \mathrm{g}$ & $3658(0.4 \%)$ & 12-27 (13.606) & 49772 & $4563(1.2 \%)$ & $12-115(14.104)$ & 64356 \\
\hline Di & $623412(76.6 \%)$ & $4-84(5.145)$ & 6415480 & 270256 (72.7\%) & $4-86(5.491)$ & 2967704 \\
\hline $\mathrm{ca} / \mathrm{tg}$ & 54064 (6.6\%) & $4-30(4.723)$ & 510666 & 22687 (6.1\%) & $4-38(4.985)$ & 226208 \\
\hline At & 148174 (18.2\%) & $4-80(5.373)$ & 1592412 & $55616(15 \%)$ & $4-86(5.385)$ & 598970 \\
\hline tc/ga & 138269 (17\%) & $4-62(4.786)$ & 1323526 & 65817 (17.7\%) & $4-64(5.417)$ & 713012 \\
\hline $\mathrm{ct} / \mathrm{ag}$ & 151296 (18.6\%) & $4-40(4.964)$ & 1502004 & 65900 (17.7\%) & $4-86(5.741)$ & 756656 \\
\hline $\mathrm{Ta}$ & 103327 (12.7\%) & $4-84(5.711)$ & 1180136 & 42285 (11.4\%) & $4-53(5.661)$ & 478768 \\
\hline $\mathrm{gt} / \mathrm{ac}$ & 24828 (3\%) & $4-31(5.534)$ & 274794 & 13993 (3.8\%) & $4-43(5.664)$ & 158526 \\
\hline $\mathrm{Cg}$ & $1667(0.2 \%)$ & $4-14(4.587)$ & 15294 & 2034 (0.5\%) & 4-19 (4.393) & 17872 \\
\hline Gc & 1787 (0.2\%) & $4-12(4.658)$ & 16648 & 1924 (0.5\%) & $4-14(4.598)$ & 17692 \\
\hline Tri & 84893 (10.4\%) & $4-81$ (4.649) & 1184076 & 49147 (13.2\%) & 4-97 (4.85) & 715137 \\
\hline tct/aga & 13187 (1.6\%) & $4-22(4.297)$ & 170001 & 4074 (1.1\%) & $4-15(4.765)$ & 58242 \\
\hline aag/ctt & 10901 (1.3\%) & $4-23(4.576)$ & 149652 & 4995 (1.3\%) & $4-26(4.826)$ & 72312 \\
\hline ttc/gaa & $9462(1.2 \%)$ & $4-21(4.521)$ & 128325 & 5545 (1.5\%) & $4-21(4.678)$ & 77823 \\
\hline att/aat & 7547 (0.9\%) & $4-55(5.427)$ & 122874 & 5545 (1.5\%) & 4-95 (5.569) & 69597 \\
\hline tta/taa & $5627(0.7 \%)$ & $4-68(5.304)$ & 89538 & $3183(0.9 \%)$ & $4-50(5.454)$ & 52083 \\
\hline ctc/gag & $4784(0.6 \%)$ & $4-16(4.438)$ & 63693 & 3635 (1\%) & $4-16(4.692)$ & 51171 \\
\hline gga/tcc & $3843(0.5 \%)$ & $4-13(4.491)$ & 51777 & 2733 (0.7\%) & 4-14 (4.643) & 38070 \\
\hline Others & 29542 (3.6\%) & $4-84(4.606)$ & 408216 & 19437 (5.2\%) & $4-97(4.737)$ & 295839 \\
\hline Tetra & 18828 (2.3\%) & $4-153$ (4.716) & 355192 & 12055 (3.2\%) & 4-96 (4.778) & 230372 \\
\hline ttta & 1014 (0.1\%) & $4-13(4.613)$ & 18712 & 845 (0.2\%) & $4-12(4.801)$ & 16228 \\
\hline tatg & 1018 (0.1\%) & $4-16(5.031)$ & 20488 & 491 (0.1\%) & $4-15(5.096)$ & 10008 \\
\hline aaat & 1550 (0.2\%) & $4-11(4.567)$ & 28316 & 954 (0.3\%) & $4-10(4.754)$ & 18140 \\
\hline aaag & 659 (0.1\%) & $4-10(4.423)$ & 11660 & 452 (0.1\%) & $4-15(4.746)$ & 8580 \\
\hline aata & 457 (0.1\%) & $4-12(4.65)$ & 8500 & 491 (0.1\%) & $4-12(4.707)$ & 9244 \\
\hline Others & 14130 (1.7\%) & $4-153$ (4.733) & 267516 & 8822 (2.4\%) & $4-96(4.766)$ & 168172 \\
\hline Penta & 6690 (0.8\%) & $4-17$ (4.379) & 146465 & 3055 (0.8\%) & $4-72$ (4.372) & 66780 \\
\hline aaaag & 356 (0.04\%) & $4-10(4.306)$ & 7665 & 173 (0.05\%) & $4-72(4.726)$ & 4085 \\
\hline aaaat & 410 (0.1\%) & $4-8(4.324)$ & 8865 & 168 (0.05\%) & $4-8$ (4.429) & 3720 \\
\hline Tttct & 194 (0.02\%) & $4-7(4.201)$ & 4075 & 79 (0.02\%) & $4-10$ (4.646) & 1835 \\
\hline Tttta & 252 (0.03\%) & $4-7$ (4.29) & 5405 & 88 (0.02\%) & $4-8$ (4.273) & 1880 \\
\hline Others & 5478 (0.7\%) & $4-17(4.398)$ & 120455 & 2547 (0.7\%) & $4-23$ (4.339) & 55260 \\
\hline Hexa & 2376 (0.3\%) & $4-41(4.37)$ & 62304 & 1251 (0.3\%) & $4-10(4.315)$ & 32388 \\
\hline
\end{tabular}

these in silico fragments, 57989 mono- (accounting for $9.37 \%$ of all primer pairs flanking mono-nucleotide motif), 475326 di(76.82\%), 67035 tri- (10.83\%), 12561 tetra- (2.14\%), 4272 penta(0.69\%), and $1579(0.26 \%)$ could produce $139830,8523025,784$ 979, 134 392, 73 686, and 31264 in silico amplification fragments, respectively, from the assembled genome of E. guineensis. From these results, $67.87 \%$ of primer pairs generate a single in silico PCR product, which suggests that these identified microsatellites sequences are only present in one copy in the genome of E. guineensis.

Meanwhile, among these designed primer pairs, 228056 (28.17\%) can be mapped onto the assembled chromosomes of E. guineensis. A higher proportion of single-copy microsatellites (185 621, 81.39\%) was detected in the microsatellites mapped to the assembled chromosomes of E. guineensis. Moreover, 14011 (6.14\%), 4749 (2.08\%), and 23675 (10.38\%) primer pairs could produce 2, 3 , and $>3$ in silico PCR products, respectively, from the assembled genomic sequence of E. guineensis.

\section{Conservation of Microsatellite Loci between Elaeis guineensis and Phoenix dactylifera and the Overlap with Genic Regions}

Subsequently, these 618762 primer pairs were used to in silico amplify the assembled genome of $P$. dactylifera. Only 7265 $(0.99 \%)$ of these primer pairs designed based on the flanking 


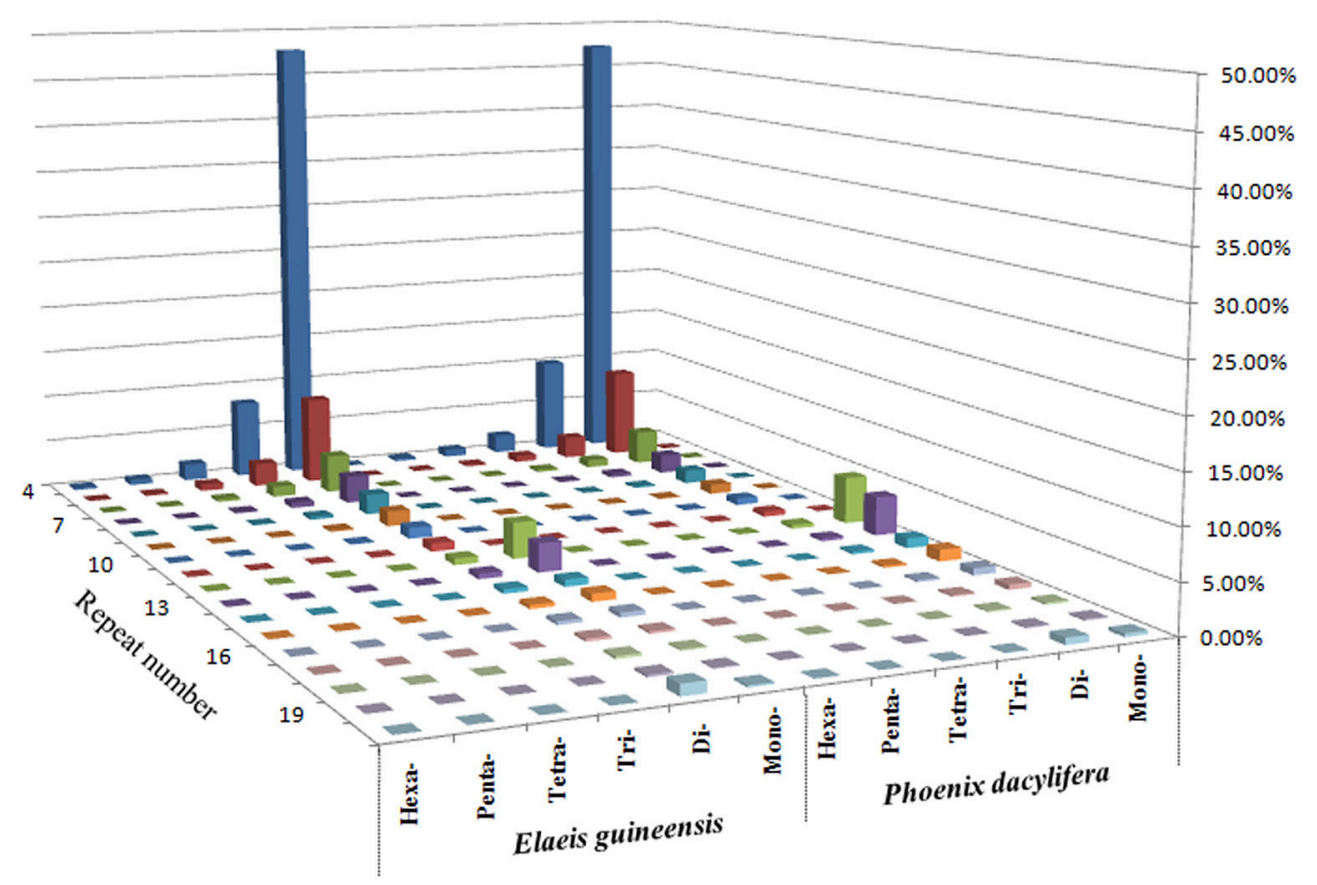

FIGURE 1 | Distribution with respect to the motif repeats of mono to hexa-nucleotide microsatellites based on the assembled genomic sequences of Elaeis guineensis and Phoenix dactylifera.

sequence of microsatellites of E. guineensis produced positive e-PCR fragments from the assembled genome of $P$. dactylifera (Table 2). Detailed information for the 7265 primer pairs is listed in Table S1. For the 7265 microsatellite markers developed from E. guineensis, 4522 (62.19\%), 1154 (15.91\%), 307 (4.22\%), and $1282(17.68 \%)$ could generate 1, 2, 3, and >3 in silico PCR products, respectively, from $P$. dactylifera. A total of 65 550 fragments were in silico amplified based on these conserved microsatellite primers between E. guineensis and $P$. dactylifera. Meanwhile, 458 mono- (accounting for $6.3 \%$ of all primer pairs flanking mono-nucleotide motif), 5443 di- (74.86\%), 1305 tri(17.95\%), 187 tetra- $(2.57 \%), 13$ penta- $(0.18 \%)$, and 12 hexa$(0.17 \%)$ primer pairs could produce 458, $56328,1961,6961$, 40 , and 802 in silico amplification fragments, respectively, from the assembled genome of $P$. dactylifera.

Among these conserved microsatellite primer pairs, a total of 4638 primer pairs could be mapped into the assemble chromosomes of E. guineensis. A total of 41710 fragments were in silico amplified based on these conserved microsatellite primers between E. guineensis and P. dactylifera, with an average of 8.99 fragments per primer. A higher proportion of primer pairs (3260, $70.29 \%$ ) only generated a single in silico PCR product in the assembled chromosomes of E. guineensis. Moreover, 335 (7.45\%), $28(0.62 \%)$, and $24(0.54 \%)$ could generate $1,2,3$, and $>3$ in silico PCR products, respectively, from the assembled genomic sequence of $P$. dactylifera.

The position of the conserved microsatellites in the assembled genome of E. guineensis was analyzed to confirm if they were located within genic or intergenic regions. The analysis results showed that the majority of the conserved microsatellites
(71.45\%) were located within genic regions, while the others (28.55\%) were located within intergenic regions (Figure S3). In different chromosomes of E. guineensis, the percentage of conserved microsatellites within genic regions varied from $54.04 \%$ in chromosome 11 to $89.41 \%$ in chromosome 13 . Of these conserved microsatellites in genic regions, $5.5 \%$ were located in untranslated regions (UTRs), which may be associated with gene expression and splicing; $28.4 \%$ were located in coding regions (CDS), which may be directly associated with amino sequences; and the remaining $66.1 \%$ were located in intron regions of genes. The analysis results suggested that these conserved microsatellites are prone to be distributed within genic regions.

\section{Cross-Genera Transferability of these Conserved Microsatellites in the Palm Species}

We chose conserved microsatellite markers that could be detected in both the assembled genomes of $P$. dactylifera and E. guineensis for transferability analysis in Palmae species. One hundred and thirty five primer pairs were selected stochastically from the 7265 conserved microsatellite markers: 93 single-copy microsatellites were selected, 19 microsatellites present in two copies were selected, 5 microsatellites present in three copies were selected, and the remaining microsatellites were present in more than three copies. The 135 primer pairs were used to amplify genomic DNA from 32 palm species. In 106 (78.5\% of cases), PCR products could be amplified in the genomic DNA of $E$. guineensis. The remaining 29 primer pairs were excluded from 


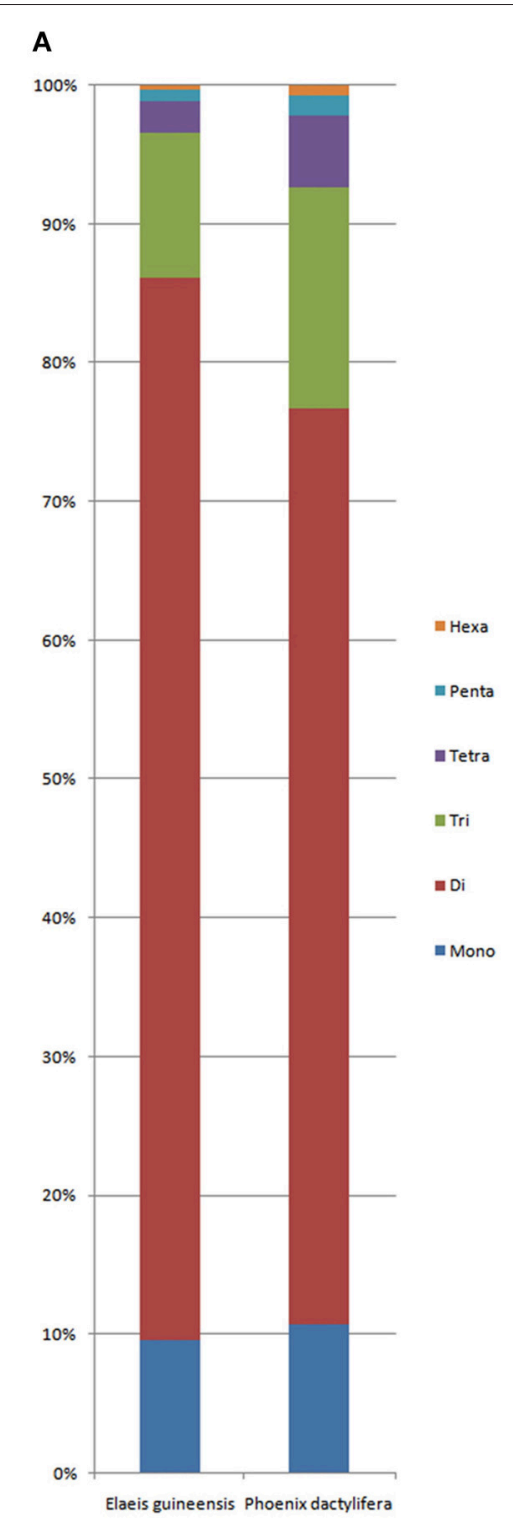

\section{B}

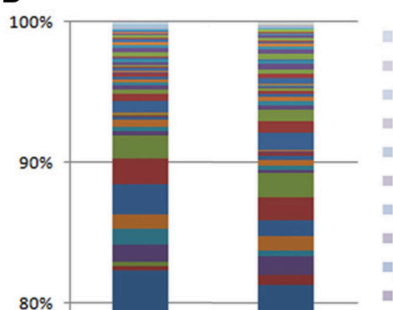

C

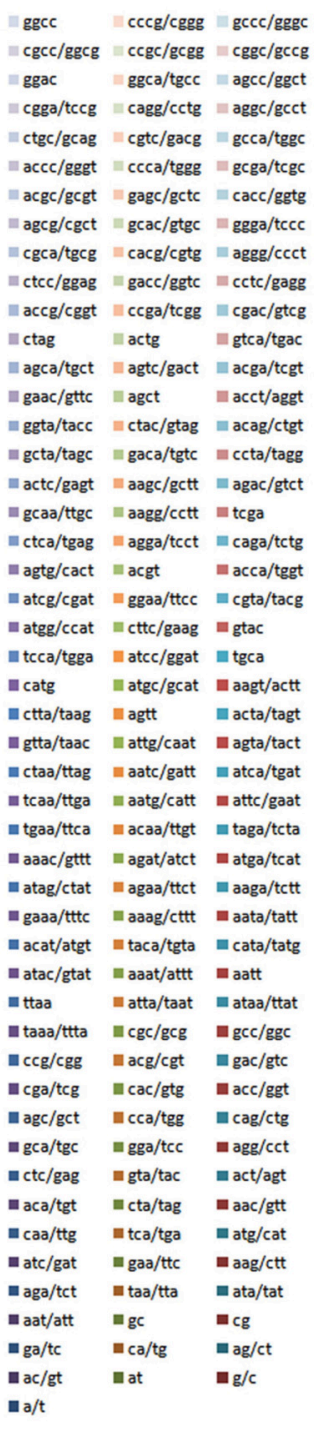

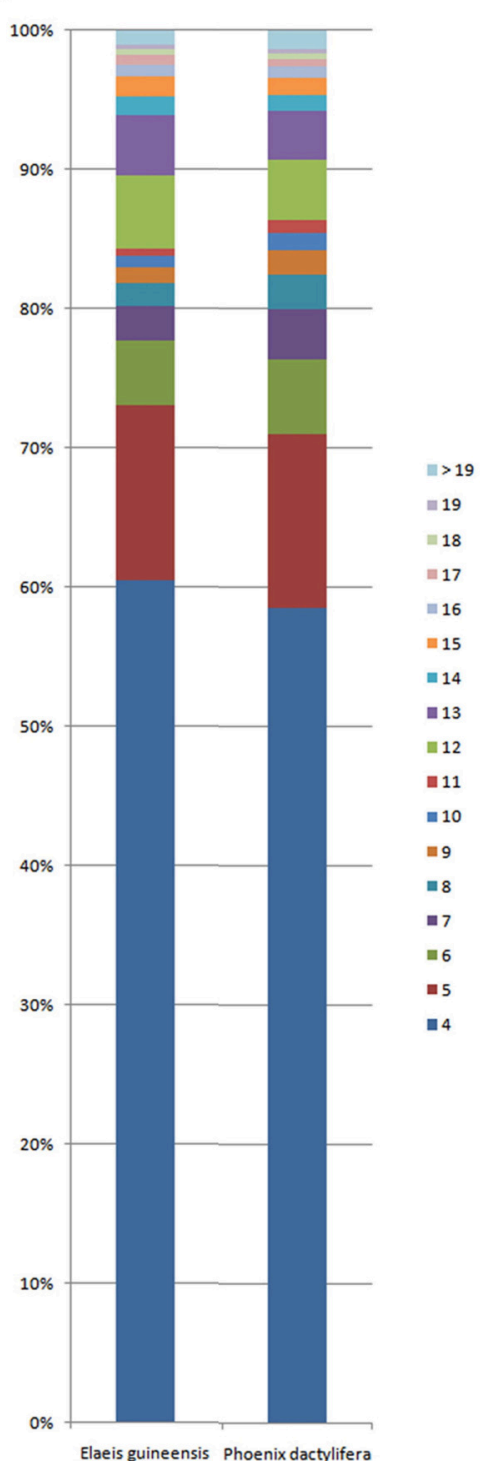

FIGURE 2 | Distribution with respect to the motif length (A), motif types (B) and repeat number (C) of microsatellites in the assembled genomes of Elaeis guineensis and Phoenix dactylifera. The vertical axis represents the percentage of SSRs with different motif lengths, motif types and repeat numbers.

further analysis due to lack of PCR products or due to weak amplification. A total of 328 alleles were detected, with an average of 3.04 alleles per microsatellite marker (Table S2). Meanwhile, these microsatellite markers also showed high transferability to other palm species, ranging from amplification in 17 to $93 \%$ of species samples with an average of $73 \%$ (Figure S4). These results indicated that these microsatellite loci were conserved in the palm species.

\section{DISCUSSION}

Although the genomic sequences of E. guineensis and $P$. dactylifera have been released, there are still no related documents which elucidate and compare the frequency and distribution of microsatellites in the two palm species. To our knowledge, this study is the first report on genome-wide identification and comparison of microsatellite loci based on the assembled genomes of E. guineensis and P. dactylifera. In previous studies, microsatellites were generally developed based on the released transcriptome data from E. guineensis and $P$. dactylifera. Tranbarger et al. (2012) detected 465 microsatellites within 2652 262 bp of E. guineensis EST sequences. Zhao et al. (2012) detected 5981 microsatellites from 28889 assembled EST sequences from $P$. dactylifera. However, our study was based on the entire assembled genomes of E. guineensis and $P$. dactylifera, including non-coding sequences. This allowed a far greater number of microsatellites (814 383 and 371629 respectively) to be identified 
TABLE 2 | The density of microsatellites, genes and transposable elements (TEs) in the chromosomes of Elaeis guineensis.

\begin{tabular}{|c|c|c|c|c|c|}
\hline & $\begin{array}{l}\text { SSR density } \\
\qquad(/ \mathrm{Mb})\end{array}$ & $\begin{array}{c}\text { Gene density } \\
\text { (/Mb) }\end{array}$ & $\begin{array}{l}\text { TE density } \\
\text { (/Mb) }\end{array}$ & $\begin{array}{l}\text { Relation coefficient between } \\
\text { SSR and TEs }\end{array}$ & $\begin{array}{c}\text { Relation coefficient between } \\
\text { SSR and genes }\end{array}$ \\
\hline Chromosome 1 & 723.71 & 39.30 & 37.96 & -0.51 & 0.75 \\
\hline Chromosome 2 & 707.23 & 36.52 & 43.29 & -0.43 & 0.65 \\
\hline Chromosome 3 & 683.53 & 34.58 & 39.94 & -0.35 & 0.82 \\
\hline Chromosome 4 & 659.76 & 30.01 & 39.58 & -0.34 & 0.69 \\
\hline Chromosome 5 & 670.82 & 36.57 & 44.15 & -0.60 & 0.84 \\
\hline Chromosome 6 & 593.98 & 29.73 & 45.31 & -0.45 & 0.83 \\
\hline Chromosome 7 & 676.89 & 32.47 & 42.30 & -0.44 & 0.74 \\
\hline Chromosome 8 & 674.58 & 35.67 & 41.22 & -0.39 & 0.85 \\
\hline Chromosome 9 & 595.82 & 28.80 & 42.72 & -0.13 & 0.80 \\
\hline Chromosome 10 & 724.40 & 38.76 & 38.32 & -0.29 & 0.78 \\
\hline Chromosome 11 & 630.33 & 32.36 & 42.14 & -0.40 & 0.86 \\
\hline Chromosome 12 & 731.01 & 39.72 & 41.56 & -0.68 & 0.84 \\
\hline Chromosome 13 & 701.11 & 34.87 & 43.53 & -0.69 & 0.85 \\
\hline Chromosome 14 & 690.89 & 38.56 & 41.26 & -0.26 & 0.71 \\
\hline Chromosome 15 & 696.67 & 35.83 & 44.92 & -0.19 & 0.80 \\
\hline Chromosome 16 & 735.56 & 38.00 & 47.54 & -0.39 & 0.73 \\
\hline
\end{tabular}

compared to previous studies (Tranbarger et al., 2012; Zhao et al., 2012; Aberlenc-Bertossi et al., 2014).

Previously, the frequency of microsatellites was reported to be very similar in different species of the same genera (Shi et al., 2013). In this study, E. guineensis and P. dactylifera are classified into different genera in the Palmae family. However, the density of microsatellites in the assembled genomic sequences of $E$. guineensis (770.4 microsatellites per $\mathrm{Mb}$ ) and P. dactylifera (733 microsatellites per $\mathrm{Mb}$ ) is also nearly identical, indicating highly similar genomic structure in the two palm species. Meanwhile, the distribution with respect to the motif type and repeat number of microsatellites in the assembled genomic sequences of the two palm species is also very similar. More specifically, $\mathrm{A} / \mathrm{T}$ rich motifs are generally dominant/major, while $\mathrm{C} / \mathrm{G}$ rich motifs are absent/scarce, which is in accordance with reported results by Shi et al. (2013). Microsatellite expansion and variation is thought to result from replication slippage by mismatch during DNA replication (Tautz and Schlotterer, 1994; Klintschar et al., 2004; Forster et al., 2015), which occurs more readily in A/T rich motifs than in $\mathrm{C} / \mathrm{G}$ rich motifs. Overall, the microsatellite distribution characteristics in the assembled genomics of the two palm species are very similar. This suggests that the two Palmae species have similar genome structure, which may be due to their relatively recent divergence (65 Myr) from a common ancestor (Singh et al., 2013).

In this study, a total of 814383 and 371629 monoto hexanucleotide repeat microsatellites were identified in the assembled genomic sequences of E. guineensis and P. dactylifera, respectively, with frequencies of 770.4 and 733 microsatellites per $\mathrm{Mb}$ or one microsatellite per 1.29 and 1.36. The microsatellite density in the assembled genomic sequences of the two Palmae species is higher than the SSR density in some species (Tautz and Schlotterer, 1994; Klintschar et al., 2004; Song et al., 2015). To our knowledge, this study is the first report on genome-wide identification of microsatellite loci in palm family. Most (99.29 and $98.39 \%$ for E. guineensis and P. dactylifera) of the identified microsatellite loci are novel compared to previous research in these two palm species (Zhao et al., 2012; Xiao et al., 2014). Although further validation may still be required, these newly developed microsatellite loci will be useful for future molecular marker studies in E. guineensis and P. dactylifera. Possible applications include the construction of high-density linkage maps, QTL fine mapping and genome-wide association mapping.

A great deal of research has been performed to identify genic microsatellites via in silico analysis of publicly available unique transcriptome sequences in E. guineensis (Tranbarger et al., 2012; Xiao et al., 2014). However, due to the lack of genomic sequences, the distribution and physical position of these publicly available microsatellite markers is still unclear, which has a negative effect on their effective utilization. In this study, we describe the genome distribution and the accurate physical position of these newly developed microsatellite markers based on the mapped sequence scaffolds of E. guineensis. The highdensity microsatellite markers with accurate physical position could be very useful for rapid selection of trait-associated markers and subsequent application of molecular breeding in E. guineensis. Previous studies have showed that the distribution of microsatellites is preferentially associated with non-repetitive DNA/gene sequences in plant genomes (Morgante et al., 2002; Gemayel et al., 2010). In our study, the genomic distribution of microsatellites was also positively correlated to that of genes but negatively correlated to that of TEs. The high accordance between the genomic distribution of microsatellites and genes may suggest a putative role of microsatellite in regulating gene function ( $\mathrm{Li}$ et al., 2002, 2004; Gemayel et al., 2010). Some microsatellite markers could also be used as functional markers to tag genes and for molecular breeding. 


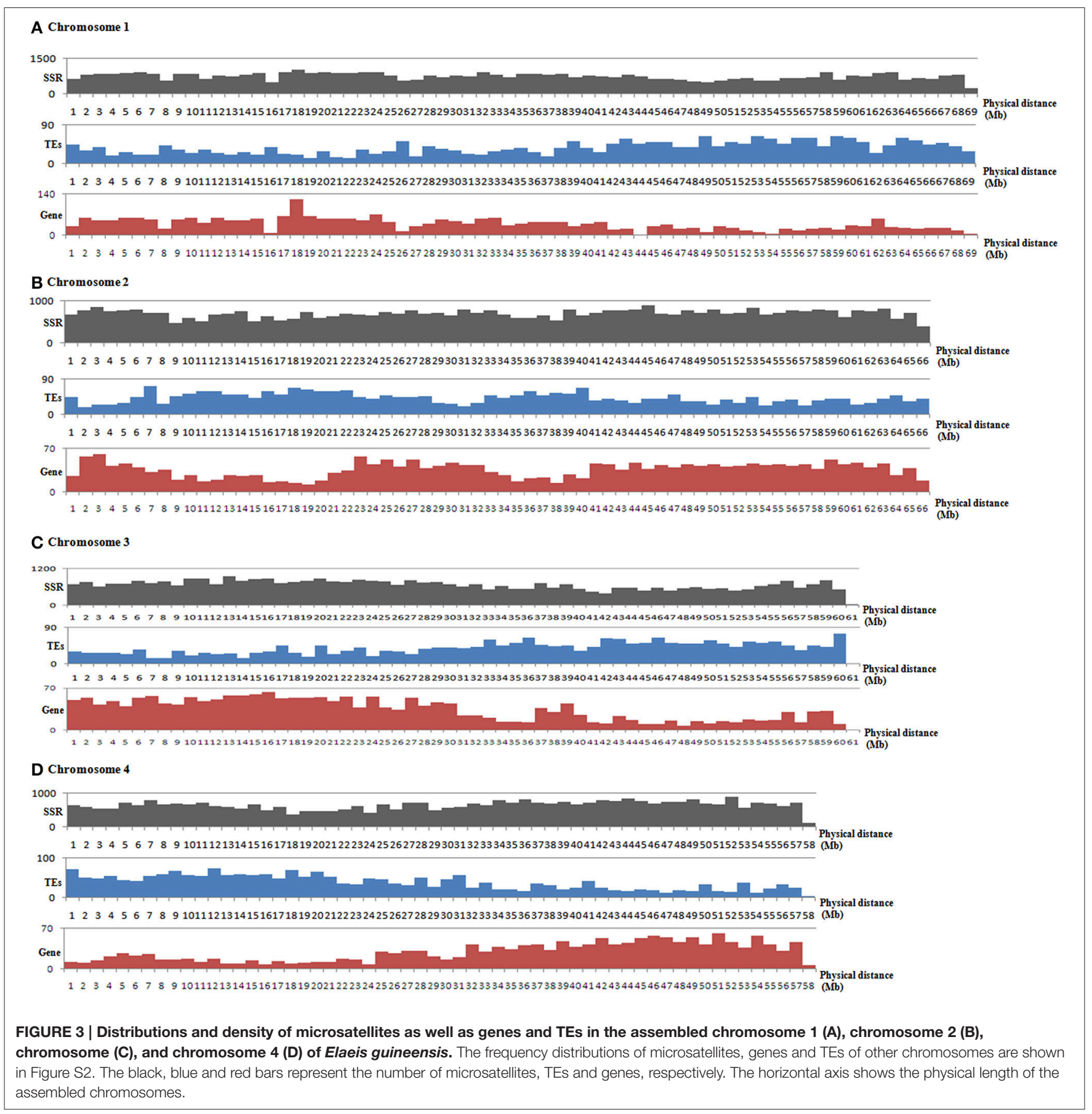

Microsatellite markers obtained from one plant species are seldom used directly in populations of closely related species. In order to screen cross-genera transferability of microsatellite markers, primer pairs flanking microsatellites from one plant species are generally used to amplify DNA from other species in the same genera (Chagne et al., 2004; Guo et al., 2006). This method has comparatively low efficiency. Normally, only a few cross-species transferable microsatellite markers can be obtained through the selection of a large number of primer pairs. This study was conducted to select cross-genera-transferable microsatellite markers in the Palmae family. In order to increase the selection efficiency, a large number of conserved microsatellites were first identified between $E$. guineensis and $P$. dactylifera. Most of these conserved microsatellites identified in the present study were also validated to have high transferability ratio in palm species in the study. In conclusion, the conserved microsatellites identified in the study will provide some genetic resources for the assessment of genetic 
diversity, the analysis of population structure, and conservation of palm species, especially for endangered species.

Electronic PCR (e-PCR) is a useful tool for simulating the process of Polymerase Chain Reaction (PCR) and for detecting potential amplification products. In the present study, validating transferability for 618762 primer pairs would be prohibitively time-consuming. Electronic PCR (e-PCR) simplified the process. The selection of parameters is important for the reliability of electronic PCR (e-PCR). The default parameters of the software are 2 bp mismatch, 1 bp gap, and 500-1000 bp product size. In the present study, in order to increase the reliability of the electronic PCR results, more stringent parameters were applied: a 1 bp mismatch and 0 bp gap were allowed in order to decrease the chance of false positives, although this may also increase the false negative rate.

Location of microsatellites in non-coding regions allows easier accumulation of mutations over the generations, and gives rise to increased variability which can be used for DNA markers. However, other microsatellites may be located in the UTR of mRNA, promoter regions of expressed genes, or even directly in CDS of genes. In such cases, microsatellite mutation may lead to phenotypic changes. Microsatellite variation in CDS can lead directly to functional protein changes, while microsatellite variation occurring in $5^{\prime}$ UTR (5'-UTRs) may affect transcription and translation, and microsatellite variation in $3^{\prime}$-UTRs may affect splicing. Therefore, these microsatellites associated with biological functions due to their location may be more highly conserved between different genotypes and even between different species, as most novel mutations are detrimental. In this study, some microsatellites were found to be conserved between palm species, and the position of these conserved microsatellites in the genome of E. guineensis was also analyzed. The majority of the conserved microsatellites $(71.45 \%)$ were located within genic regions, while the remainder $(28.55 \%)$ were located within intergenic regions. It is possible that some conserved microsatellites in intergenic regions were located on promoter regions of genes, such that mutation of these microsatellites may lead to a change in gene expression. Mutation of the $71.45 \%$ of conserved microsatellites located in genic regions may directly lead to changes in amino sequence, splicing, and gene expression. In summary, microsatellites associated with biological function due to their genomic location in genic regions may be more conserved between different genotypes and even species, and hence more transferable to other species. Therefore, selection of genic microsatellites may be a useful strategy for identifying high proportions of markers transferable between species.

\section{REFERENCES}

Aberlenc-Bertossi, F., Castillo, K., and Tranchant-Dubreuil, C. (2014). In silico mining of microsatellites in coding sequences of the date palm (Arecaceae) genome, characterization, and transferability. Appl. Plant Sci. 2:1300058. doi: 10.3732/apps. 1300058

AI-Dous, E., George, B., AI-Mahmoud, M. E., AI-Jaber, M. Y., Wang, H., Salameh, Y. M., et al. (2011). De novo genome sequencing and comparative

\section{CONCLUSIONS}

In the last two decades, advances in next-generation sequencing technology have facilitated the development of molecular markers. In this study, genome-wide identification of microsatellites was conducted based on the assembled genomic sequences of E. guineensis and $P$. dactylifera. A high density of microsatellites was identified in the two species, which will provide a useful tool for research into genetic diversity, gene mapping and molecular breeding in these species.

It has been previously documented that some microsatellites could be transferable between species or even between genera (Chagne et al., 2004; Mnejja et al., 2005; Guo et al., 2006; Pandey et al., 2013), In this study, we tried to develop a large number of conserved microsatellites which can be used for genetic analyses in different palm species. A total of 7265 conserved microsatellite markers were identified between E. guineensis and P. dactylifera. In the future, these identified conserved SSR markers may be regarded as useful genetic resources for diversity conservation in different species in the Palmae family, especially in endangered species.

\section{AUTHOR CONTRIBUTORS}

YX and WX did the DNA extract and subsequently PCR amplification, participated in the design of the study, performed the statistical analysis and drafted the manuscript. JM did the extraction of DNAs and PCR amplification. AM critically revised the manuscript. XL, HF, ZM, and MP participated in the design of the study. All authors read the final manuscript and have given final approval of the version to be published.

\section{FUNDING}

This work was supported by the Natural Science Foundation of China (No.31301358), the Scientific and Technological Cooperation Projects of Hainan province (No.SQ2015GJXM0076), National Nonprofit Institute Research Grant of CATAS-ITBB (No.1630052015050), and The Major Technology Project of Hainan (ZDZX2013023-1).

\section{SUPPLEMENTARY MATERIAL}

The Supplementary Material for this article can be found online at: http://journal.frontiersin.org/article/10.3389/fpls.2016. 01578

genomics of date palm (Phoenix dactylifera). Nat. Biotechnol. 29, 521-527. doi: $10.1038 /$ nbt. 1860

AI-Mssallem, I. S., Hu, S., Zhang, X., Lin, Q., Liu, W., Tan, J., et al. (2013). Genome sequence of the date palm Phoenix dactylifera L. Nat. Commun. 4, 2274. doi: $10.1038 /$ ncomms 3274

Bohra, A., Dubey, A., Saxena, R. K., Penmetsa, R. V., Poornima, K. N., Kumar, N., et al. (2011). Analysis of BAC-end sequences (BESs) and development of BES-SSR markers for genetic mapping and hybrid purity assessment in 
pigeonpea (Cajanus spp.). BMC Plant Biol. 11:56. doi: 10.1186/1471-222911-56

Chagné, D., Chaumeil, P., Ramboer, A., Collada, C., Guevara, A., Cervera, M. T., et al. (2004). Cross-species transferability and mapping of genomic and cDNA SSRs in pines. Theor. Appl. Genet. 109, 1204-1214. doi: 10.1007/s00122-0041683-z

Decroocq, V., Favé, M. G., Hagen, L., Bordenave, L., and Decroocq, S. (2003). Development and transferability of apricol and grape EST microsatellite markers across taxa. Theor. Appl. Genet. 106, 912-922. doi: 10.1007/s00122002-1158-Z

Forster, P., Hohoff, C., Dunkelmann, B., Schürenkamp, M., Pfeiffer, H., Neuhuber, F., et al. (2015). Elevated germline mutation rate in teenage fathers. Proc. R. Soc. B 282, 1803. doi: 10.1098/rspb.2014.2898

Gasic, K., Han, Y., Kerbundit, S., Shulaev, V., Lezzoni, A. F., Stover, E. W., et al. (2009). Characteristics and transferability of new apple EST-derived SSRs to other Rosaceae species. Mol. Breed. 23, 397-411. doi: 10.1007/s11032-0089243-x

Gemayel, R., Vinces, M. D., Legendre, M., and Verstrepen, K. J. (2010). Variable tandem repeats accelerate evolution of coding and regulatory sequences. Annu. Rev. Genet. 44, 445-477. doi: 10.1146/annurev-genet-072610-155046

Guo, W., Wang, W., Zhou, B., and Zhang, T. (2006). Cross-species transferability of G. arboretum-derived EST-SSRs in the diploid species of Gossypium. Theor. Appl. Genet. 112, 1573-1581. doi: 10.1007/s00122-006-0261-y

Klintschar, M., Dauber, E. M., Ricci, U., Cerri, N., Immel, U. D., Kleiber, M., et al. (2004). Haplotype studies support slippage as the mechanism of germline mutations in short tandem repeats. Electrophoresis 25, 3344-3348. doi: 10.1002/elps.200406069

Li, Y. C., Korol, A. B., Fahima, T., Beiles, A., and Nevo, E. (2002). Microsatellites: genome distribution, putative functions and mutational mechanisms: a review. Mol. Ecol. 11, 2453-2465. doi: 10.1046/j.1365-294X.2002.01643.X

Li, Y. C., Korol, A. B., Fahima, T., and Nevo, E. (2004). Micosatellites within genes: structure, fuction, and evolution. Mol. Biol. Evol. 21, 991-1007. doi: 10.1093/ molbev/msh073

Low, E. T., Alias, H., Boon, S. H., Shariff, E. M., Tan, C. Y. A., Ooi, L. C. L., et al. (2008). Oil palm (Elaeis guineensis Jacq.) tissue culture ESTs: identifying genes associated with callogenesis and embryogenesis. BMC Plant Biol. 8:62. doi: 10.1186/1471-2229-8-62

Marek, L. F., Mudge, J., Darnielle, L., Grant, D., Hanson, N., Paz, M., et al. (2001). Soybean genomic survey: BAC-end sequences near RFLP and SSR markers. Genome 44, 572-581. doi: 10.1139/g01-052

Mnejja, M., Garcia-Mas, J., Howad, W., and Arus, P. (2005). Development and transportability across Prunus species of 42 polymorphic almond microsatellites. Mol. Ecol. Notes 5, 531-535. doi: 10.1111/j.1471-8286. 2005.00977.x

Mnejja, M., Garcia-Mas, J., Audergon, J. M., and Arus, P. (2010). Prunus microsatellite marker transferability across rosaceous crops. Tree Genet. Genomes 6, 689-700. doi: 10.1007/s11295-010-0284-z

Morgante, M., Hanafey, M., and Powell, W. (2002). Microsatellite are preferentially associated with nonrepetitive DNA in plant genomes. Nat. Genet. 30, 194-200. doi: $10.1038 / \mathrm{ng} 822$

Pandey, G., Misra, G., Kumari, K., Gupta, S., Parida, S. K., Chattopadhyay, D., et al. (2013). Genome-wide development and use of microsatellite markers for largescale genotyping applications in Foxtail millet [Setaria italic (L.)]. DNA Res. 20, 197-207. doi: 10.1093/dnares/dst002

Qiu, D., Morgan, C., Shi, J., Long, Y., Liu, J., Li, R., et al. (2006). A comparative linkage map of oilseed rape and its use for QTL analysis of seed oil and erucic acid content. Theor. Appl. Genet. 114, 67-80. doi: 10.1007/s00122-006-0411-2

Rivera, R., Edwards, K. J., Barker, J. H. A., Arnold, G. M., Ayad, G., Hodgkin, T., et al. (1999). Isolation and characterization of polymorphic microsatellites in Cocos nucifera L. Genome 42, 668-675. doi: 10.1139/g98-170

Rotmistrovsky, K., Jang, W., and Schuler, G. D. (2004). A web server for performing electronic PCR. Nucleic Acids Res. 32, W108-W112. doi: 10.1093/nar/gkh450

Rozen, S., and Skaletsky, H. (1999). "Primer3 on the WWW for general users and for biologist programmers," in Bioinformatics Methods and Protocols, eds S. Misener and S. A. Kraweiz (New York, NY: Springer), 365-386.
Schuler, G. D. (1997). Sequence mapping by electronic PCR. Genome Res. 7, 541-550.

Shi, J., Huang, S., Zhan, J., Yu, J., Wang, X., Hua, W., et al. (2013). Genome-wide microsatellite characterization and marker development the sequenced Brassica crop species. DNA Res. 21, 53-68. doi: 10.1093/dnares/dst040

Singh, R., Ong-Abdullah, M., Low, E. T., Manaf, M. A., Rosli, R., Nookiah, R., et al. (2013). Oil palm genome sequence reveals divergence of interferfertile species in Old and New worlds. Nature 500, 335-339. doi: 10.1038/nature12309

Smit, A. F. A., Hubley, R., and Green, P. (2013/2015). RepeatMasker Open-4.0. Available online at: http://www.repeatmasker.org

Song, X., Ge, T., Li, Y., and Hou, X. (2015). Genome-wide identification of SSR and SNP markers from the non-heading Chinese cabbage for comparative genomic analysis. BMC Genomics 16:328. doi: 10.1186/s12864-015-1534-0

Stewart, C. N. Jr., and Via, L. E. (1993). A rapid CTAB DNA isolation technique useful for RAPD fingerprinting and other PCR application. Biotechnique 14, 748-749.

Tautz, D., and Schlotterer, C. (1994). Simple sequence. Curr. Opin. Genet. Dev. 4, 832-837. doi: 10.1016/0959-437X(94)90067-1

Thurston, M., and Field, D. (2005). Msatfinder: Detection and Characterization of Microsatellites. Oxford: CEH. Available online at: https://github.com/knirirr/ Msatfinder

Tranbarger, T. J., Kluabmongkol, W., and Sangsrakru, D. (2012). SSR markers in transcripts of gene linked to post-transcriptional and transcriptional regulatory functions during vegetative and reproductive development of Elaeis guineensis. BMC Plant Biol. 12:1. doi: 10.1186/1471-2229-12-1

Wang, H., Walla, J. A., Zhong, S., Huang, D., and Dai, W. (2012). Development and cross-species/genera transferability of microsatellite markers discovered using 454 genome sequencing in chokecherry (Prunus virginiana L.). Plant Cell Rep. 31, 2047-2055. doi: 10.1007/s00299-012-1315-Z

Xia, W., Xiao, Y., Liu, Z., Luo, Y., Mason, A. S., Fan, H., et al. (2014). Development of gene-based simple sequence repeat markers for association analysis in Cocos nucifera. Mol. Breed. 34, 525-535. doi: 10.1007/s11032-014-0055-x

Xiao, Y., Chen, L., Zou, J., Tian, E., Xia, W., and Meng, J. (2010). Development of a population for substantial new type Brasscia napus diversified at both A/C genomes. Theor. Appl. Genet. 121, 1141-1150. doi: 10.1007/s00122-010-1378-6

Xiao, Y., Zhou, L., Xia, W., Mason, A. S., Yang, Y., Ma, Z., et al. (2014). Exploiting transcriptome data for the development and characterization of gene-based SSR markers related to cold tolerance in oil palm (Elaeis guineensis). BMC Plant Biol. 14:384. doi: 10.1186/s12870-014-0384-2

Xu, J., Qian, X., Wang, X., Li, R., Cheng, X., Yang, Y., et al. (2010). Construction of an integrated genetic linkage map for the A genome of Brassica napus using SSR markers derived from sequenced BACs in B. rapa. BMC Genomics 11:594. doi: 10.1186/1471-2164-11-594

Zhang, Q., Ma, B., Li, H., Chang, Y., Han, Y., Li, J., et al. (2012). Identification, characterization, and utilization of genome-wide simple sequence repeats to identify a QTL of acidity in apple. BMC Genomics 13:537. doi: 10.1186/14712164-13-537

Zhao, Y., Williams, R., Prakash, C. S., and He, G. (2012). Identification and characterization of gene-based SSR markers in date palm (Phoenix dactylifera L.). BMC Plant Biol. 12:237. doi: 10.1186/1471-2229-12-237

Conflict of Interest Statement: The authors declare that the research was conducted in the absence of any commercial or financial relationships that could be construed as a potential conflict of interest.

The reviewer CZ declared a shared affiliation, though no other collaboration, with several of the authors ZM, MP to the handling Editor, who ensured that the process nevertheless met the standards of a fair and objective review.

Copyright (c) 2016 Xiao, Xia, Ma, Mason, Fan, Shi, Lei, Ma and Peng. This is an open-access article distributed under the terms of the Creative Commons Attribution License (CC BY). The use, distribution or reproduction in other forums is permitted, provided the original author(s) or licensor are credited and that the original publication in this journal is cited, in accordance with accepted academic practice. No use, distribution or reproduction is permitted which does not comply with these terms. 